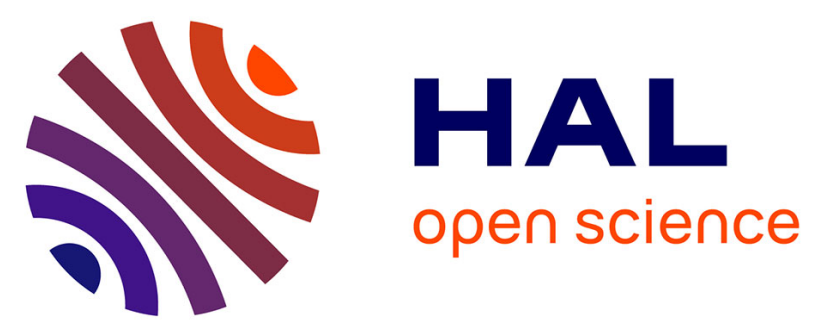

\title{
Long lasting breeding performance differences between wild-born and released femalesin a reinforced North African Houbara bustard (Chlamydotis undulata undulata) population: amatter of release strategy
} Leo Bacon, Alexandre Robert, Yves Hingrat

\section{To cite this version:}

Leo Bacon, Alexandre Robert, Yves Hingrat. Long lasting breeding performance differences between wild-born and released femalesin a reinforced North African Houbara bustard (Chlamydotis undulata undulata) population: amatter of release strategy. Biodiversity and Conservation, 2019, 28 (3), pp.553-570. 10.1007/s10531-018-1651-6 . mnhn-02553975

\section{HAL Id: mnhn-02553975}

https://hal-mnhn.archives-ouvertes.fr/mnhn-02553975

Submitted on 24 Apr 2020

HAL is a multi-disciplinary open access archive for the deposit and dissemination of scientific research documents, whether they are published or not. The documents may come from teaching and research institutions in France or abroad, or from public or private research centers.
L'archive ouverte pluridisciplinaire HAL, est destinée au dépôt et à la diffusion de documents scientifiques de niveau recherche, publiés ou non, émanant des établissements d'enseignement et de recherche français ou étrangers, des laboratoires publics ou privés. 
1 Long lasting breeding performance differences between wild-born and

2 released females in a reinforced North African Houbara bustard

3 (Chlamydotis undulata undulata) population: a matter of release strategy.

4 Léo Bacon ${ }^{\mathrm{a}, \mathrm{b},{ }^{*},{ }^{*},}$, Alexandre Robert ${ }^{\mathrm{b}}$, Yves Hingrat $^{\mathrm{a}, \mathrm{c}}$

$5 \quad{ }^{a}$ Emirates Center for Wildlife Propagation, PO Box 47, 33250 Missour, Morocco.

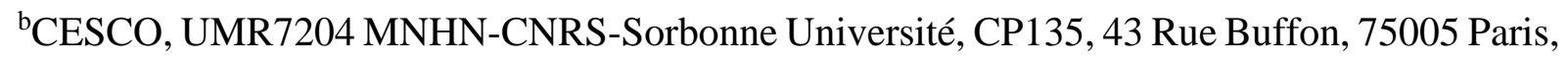

7 France.

${ }^{\mathrm{c}}$ Reneco International Wildlife Consultants LLC., Po Box 61741, Abu Dhabi, U.A.E.

*Corresponding author (bacon.leo@gmail.com)

***orcid ID: 0000-0003-1200-9902

\section{Abstract}

The success of translocation programmes is reflected by the ability of translocated individuals to survive and reproduce in their new environment. However, it has previously been reported that translocated individuals have lower demographic performance than their wild-born conspecifics, due to management and individual factors (such as release conditions or age). Here, we study six breeding parameters in free-ranging females of the North African Houbara bustard (Chlamydotis undulata undulata) and compare these parameters between captive-bred released $(n=204)$ and wild-born $(n=101)$ birds, considering the age of individuals and the period of release (autumn versus spring). Our results indicate that (1) captive-bred released females successfully breed in the wild; (2) for three out of the six breeding parameters studied, released females show lower performances than wild-born females; but, (3) Although we observed consistently reduced breeding performances in one year old females relative to older females, 
we did not uncover any interaction between age and the origin of females, suggesting that the impairment of breeding parameters in released females is long lasting; and, (4) interestingly, this impairment of breeding parameters depends on the period of release, with lower breeding performances for spring releases compared to autumn releases. Overall, our study highlights the capacity of captive-bred females to reproduce in the wild, contributing to the dynamics of the population beyond their individual history. Our results also uncover complex variations of breeding parameters in translocated birds, but suggest that these differences can be minimized through an appropriate translocation strategy.

Key-words: Captive-breeding, post-release effect, reinforcement, reproduction, translocation

\section{Introduction}

The success of conservation translocation programmes is commonly defined as the capacity of translocated populations to persist without further intervention (Ewen et al. 2012, IUCN 2013). Assessment of success thus requires an understanding of the long-term dynamics and viability of translocated populations (Robert et al. 2015a), which first and foremost requires a long-term demographic assessment (Sutherland et al. 2010). However, such assessments are rarely achieved, mainly due to a lack of resources to support appropriate monitoring and difficulties associated with data analysis (Fischer \& Lindenmayer 2000, Sutherland et al. 2010). Translocated populations are complex, heterogeneous and unbalanced systems (Robert et al. 2007), and the assessment of demographic performances is required to encompass this complexity. In particular, it is necessary to assess potential demographic variations among translocated and wild-born organisms.

Previous knowledge indicates that the demography of translocated populations is likely to be affected by a variety of genetic and non-genetic issues. Genetic issues include outbreeding depression (Huff et al. 2011) and ill adaptation (Montalvo \& Ellstrand 2001) as well as 
inbreeding and drift loads (Robert 2009). In cases of translocations based on captive breeding, adaptation to captivity (Frankham 2008) might be an important additional issue. In animals, non-genetic issues are associated with captive-rearing and release strategies, leading to various factors potentially affecting demographic rates in the wild. These include individual condition, such as health status, physiology and behaviour (Champagnon et al. 2012, Dickens et al. 2010, Hardouin et al. 2014, Tavecchia et al. 2009), proximity of the release site with other populations (Mihoub et al. 2011), period of release (Hardouin et al. 2014), age at release (Sarrazin et al. 1996) or the size and composition of the animal group (Hardouin et al. 2014, 2015a). Additionally, the potential impairment of demographic performances of translocated individuals might be explained by the interaction of their phenotype with their new environment and release conditions.

Post-release effects have been defined as impairments of vital rates caused by translocation conditions (Ewen et al. 2012). Such effects can be estimated either by assessing changes in vital rates over time in a release cohort (e.g., Armstrong \& Ewen 2001, Tavecchia et al. 2009) or by comparing vital rates of translocated and resident animals over the same time period (e.g., Brown et al. 2006). A number of studies in translocated vertebrates have documented these post-release effects, mostly in terms of survival probabilities (Armstrong et al. 2017, Bertolero \& Oro 2009, Hardouin et al. 2014, Sarrazin et al. 1994) but also in reproduction rates (Bertolero \& Oro 2009, Converse et al. 2013, Sarrazin et al. 1996, Tavecchia et al. 2009) and even in dispersal behaviour (Le Gouar et al. 2008, Mihoub et al. 2011). In the particular cases of translocated individuals originating from captive-breeding, any impairments of vital rates in translocated individuals can result either from translocation conditions (i.e., post-release effects per se), captivity conditions (including genetic and non-genetic issues), or the interaction of translocation and captivity conditions. 
However, disentangling the various sources of demographic variation in translocated populations (and in particular those related to the translocation protocol from other sources of variation) is challenging because (1) the comparison between translocated and wild-born animals requires the monitoring of wild-born individuals; a difficult task often hindered by several methodological and ethical issues, and (2) changes of vital rates over time in translocated individuals can be confounded by other longitudinal sources of demographic heterogeneity, such as age effects (Bacon et al. 2017a), or by inter-individual heterogeneity (e.g., apparent improvement of survival due to the selection of the best survivors).

The North African Houbara bustard (Chlamydotis undulata undulata, hereafter Houbara - Fig. 1) is a medium-sized bird historically distributed from Northern Mauritania to Egypt. It is threatened by over-hunting, poaching and habitat degradation, which have led to the decline of the species during the second half of the twentieth century (Goriup 1997). This decline led to the establishment of a reinforcement programme (the Emirates Center for Wildlife Propagation, ECWP, see Material and Methods) which employs captive breeding and regular releases of captive-bred individuals, combined with ecological research and hunting management, in order to increase the population size of the threatened Houbara throughout its range and to maintain viable populations in adequacy with traditional Arab falconry (Lacroix et al. 2003).

Importantly, captive-bred Houbara are released as juveniles (within their first year of life) either before or during the breeding season (in autumn and in spring, respectively). Previous researches on translocated Houbara indicate that (1) captive-bred juveniles exhibit reduction in their short-term survival (the magnitude of which varies depending on the release season and meteorological conditions), compared to long-term survival (Hardouin et al. 2014), and (2) oneyear-old released females have reduced breeding performance relative to older released females (Bacon et al. 2017a). These results suggest that translocated individuals may experience changes of vital rates over time. However, in these previous assessments of demographic rates, 
potential changes caused by translocation conditions, captivity conditions and their interactions may have been confounded with age effects. Thus, a rigorous assessment of these potential captivity and translocation effects requires formal comparison between captive-bred released and wild-born individuals of different ages. Hereafter, demographic effects occurring only in the first year following release (i.e., in one-year-old individuals) are referred to as short-term differences and effects occurring independently of the time since release (i.e., in individuals of any age) are referred to as long lasting differences.

Here, we investigate potential differences in breeding performances between wild-born and released captive-bred females, while controlling for age, period of release, and temporal and individual effects. The Houbara is a ground-nesting, gyneparental incubating species. In Eastern Morocco, females breed from mid-February to mid-June. They lay generally two to three eggs, at intervals of 2.5 days, which they incubate for an average of 23 days (Gaucher 1995). In case of nest failure, females generally initiate a replacement clutch. Houbara chicks are nidifugous but still rely on their mother for food during the first 10 days after hatching (Saint Jalme \& van Heezik 1996) and fledge at a mean age of 60 days (Hardouin et al. 2012). Maximum longevity observed in the wild was at least 13 years old for a wild-born female and 10 years old for captive-bred released females (Bacon et al. 2017a). The diet of the Houbara bustard is generalist and opportunistic and present strong seasonal variation in animal (e.g. Coleoptera, Hymenoptera) and vegetal (e.g. Asteraceae, Brassicaeae, Chenopodiaceae - Bourass et al. 2012) proportion.

We used data collected from a longitudinal nest survey conducted between 2002 and 2016 in the reinforced Houbara population in Eastern Morocco.

We focused on six breeding parameters: nesting effort (number of nesting attempts per breeding season per female), nest initiation date, clutch size, egg volume, daily nest survival and brood 
survival. We hypothesized that potential short-term reduction of performance in released Houbara are related to their potentially low condition and limited experience with their new environment (Bertolero \& Oro 2007, Bertolero et al. 2009). Thus, potential differences between released and wild-born individuals should decrease with increasing age, reflecting both individual (i.e., gain in experience within an individual, Mauck et al. 2012) and populationscale processes (i.e., death of lower-quality individuals, assuming that survival and breeding performance may positively covary, Robert et al. 2015b). We thus hypothesize that the difference in breeding performances of wild-born and captive-bred released females will decrease with their age (which is confounded with the time since release for released females). In other words, we expect to find an interaction between the origin of females (released vs. wild-born) and their age.

\section{Material and methods}

\section{Study area}

The study was conducted in Eastern Morocco, in the ECWP intervention area (Fig. 2). The study area encompasses approximately $50000 \mathrm{~km}^{2}$ and is characterized by an arid climate marked by irregular rainfalls (less than $200 \mathrm{~mm} /$ year). Mean temperatures vary from $6.80^{\circ} \mathrm{C}$ in winter (December to February) to $26.64^{\circ} \mathrm{C}$ in summer (June to August). The habitat in the study area is characterized by sparse, shrubby vegetation. Vegetation cover in the plains mainly comprises Chenopodiaceae such as Salsola spp., Hamada spp. and Compositae such as Artemisia herba-alba. In clayand silt-rich areas created by seasonal runoff waters, Salsola sp. is often associated with Atriplex spp. Drainage courses and wadis are characterized by Zizyphus lotus (Ramnaceae) and Retama sp. (Leguminosae). On the high plateaux and slopes, from the extreme east to the Oran region of Algeria, the vegetation is dominated by Stipa tenacissima 
grazing. Mixed herds are generally composed of 100-300 heads of sheep and goats. Nomads

settle camps within the study area, whereas some shepherds come from surrounding villages

(Le Cuziat et al. 2005).

\section{Emirates Center for Wildlife Propagation and reinforcement programme}

The EWCP was established in 1995 to mitigate the severe decline in Houbara populations occurring in the second half of the $20^{\text {th }}$ century. Two ECWP breeding stations were built for captive breeding (in Missour in 1995 and in Enjil in 2005, see online appendix A). Detailed descriptions of the captive breeding programme can be found in Lesobre et al. (2010) and Chargé et al. (2010, 2014). From 1996 to 2016, 108486 birds were released in North Africa (94 374 in the study area). Locally, these intensive releases may induce density dependent process leading to deleterious biotic interactions (intraspecific competition, disturbance, predation) and negatively impact life history parameters (Azar et al. 2016, Bacon et al. 2017b).

Within the study area, the overall number of birds released progressively increased over the years (from 28 in 1996 to 9084 in 2016, see online appendix B), with an increase in the number of release sites and a decrease in the group size per release (Hardouin et al. 2014). The release group size varied between sites (from four to 498 individuals, median $=18$ individuals). Houbara were released at an average of $6 \pm 3$ (standard deviation) months of age in autumn (August to December), and an average of $9 \pm 1$ months of age in spring (February to May). Autumn releases were managed to avoid direct mortality and disturbance due to hunting. In addition, since 2003, some birds were released in summer (June) at an average of $3 \pm 0.6$ months of age, but they represent only $8 \%$ of total releases in the study area. As such, in this study, we only focused on captive-bred females released in spring and autumn.

Hunting was banned between 2000 and spring 2005, and subsequently restricted to the autumn/winter period (October-January) confined within an area comprising $60 \%$ of the 
intervention area. Since 2014, it is estimated that on average 2000 birds are harvested every year, with captive-bred individuals representing $85 \%$ of the hunting bags (ECWP unpublished data).

\section{Monitoring of captive-bred released females}

Prior to 2006, released Houbara were tagged on the left tarsus with an aluminium ring displaying a unique ID number. Beginning in 2006, all released individuals were subcutaneously tagged with a unique radio-frequency identification (RFID) microchip (TROVAN LID100 implantable transponder, DorsetID; see Hardouin et al. 2015b for details). In Eastern Morocco, 3014 released birds (49\% females) were equipped with transmitters (1 937 with VHF transmitters, 933 with satellite transmitters and 144 with GSM transmitters). The VHF transmitters used in this study were battery-powered necklace units with mortality signals (11 g model RI-2B-M, 20 g model RI-2D-M, Holohil System Ltd., Carp, Ontario, Canada) and solar-powered backpack (19 g model, Merlin Systems Inc., Boise, Idaho, USA). During the breeding season, females fitted with VHF transmitters were searched for at least once per week. The satellite and GSM transmitters used in this study were backpack solar-powered transmitters: PTT-100 30 or 45 g Solar GPS PTT and 30 g solar GSM (Microwave Telemetry, Inc., Columbia, Maryland, USA). To identify breeding events of individuals equipped with satellite transmitters, individuals' movements were remotely monitored. When stationary locations were recorded, the last location was checked in the field to confirm whether the individual was dead or nesting.

\section{Trapping and identification of wild-born nesting females}

Trapping of wild-born females was performed during egg-collection campaigns in the breeding season of the periods 1997-1998, 2001-2009 and 2015-2016 (see details in online appendix C). Egg collection campaigns were aimed at building the founder population of the ECWP captive- 
breeding program (Lesobre et al. 2010, Chargé et al. 2014). Females were caught using nylon snares around their nest or around their recently hatched chicks. In total, 143 wild-born females were caught while nesting and were equipped with a transmitter or transmitter replacement. In addition, 10 wild-born females captured and equipped with VHF/PTT transmitters as juveniles were found nesting in later years (see Hardouin et al. 2012 for more details of the capture and monitoring procedures).

\section{Data collection}

\section{Breeding parameters}

Six breeding parameters were assessed from breeding survey data collected from 2002-2016: the nesting effort (number of nesting attempts per breeding season per female), the clutch size, the egg volume, the nest initiation date, the daily nest survival rate and the daily brood survival rate. The number of years for which survey data was available varied slightly between breeding parameters, this is detailed in online appendix D.

\section{- Nest survey}

Nests were located from a collaborative survey with shepherds and from individually tracked females. Because of the bias inherent to the trapping and egg-collection campaigns (all wildborn non-equipped nesting females had their eggs collected if not already hatched and were caught) and to avoid confounding factors between transmitter presence and female origin (wildborn and captive-bred), we only selected those nests for which the nesting female was equipped with a transmitter at nest initiation. In total, 703 nests from 305 females were selected for this study. For wild-born females, 300 nests were from 91 wild-born females first captured on their nest, and 21 were from 10 females captured and marked at the juvenile stage. For captive-bred females $(\mathrm{n}$ nests $=382$ ), we selected nests from cohorts of females that were released in their 
first year of life from August to November (in autumn, before the breeding season, $\mathrm{n}$ nests $=$ 122, $\mathrm{n}$ females $=74$ ) and from February to May (in spring, during the breeding season, $\mathrm{n}$ nests $=260, \mathrm{n}$ females $=130)$. Nests were visited on average every $6 \pm 3.60$ days. In addition, since 2013, camera traps were deployed at some of the monitored nests $(n=45)$, providing a continuous survey effort and more accurate assessments of nest fate. Nests equipped with camera traps were visited on average every $8 \pm 5.07$ days. Preliminary unpublished results did not highlight any deleterious effects of the camera trap monitoring on nest survival in our study area (Bacon 2017a). The use of camera traps gave great details on the guild of species predating on Houbara nests. It is composed of the red fox (Vulpes vulpes), the golden jackal (Canis aureus), stray dogs (Canis lupus familiaris), desert hedgehog (Paraechiinus aetiopicus), common raven (Corvus corax) and brown-necked raven (Corvus ruficollis). Nest failure was recorded for a visit interval if the clutch disappeared without signs of hatching (Mabee 1997) or was abandoned. We considered a nest to have survived if at least one egg hatched (Mayfield 1975).

The clutch size was defined as the maximum number of eggs observed in the nest. Since 2003, the length, width (to the nearest hundredth of a centimetre) and mass (to the nearest tenth of a gram) of each egg were measured. The width and length of eggs were then used to calculate the egg volume (Vol) in $\mathrm{cm}^{3}$ (see details in Bacon et al. 2017a). The nest initiation date was estimated from the calculation of the eggs' incubation stage (in days) at the time of measurement (based on the egg weight loss equation from Hoyt 1979, see details in Bacon et al. 2017a).

The nesting effort was calculated as the number of nests initiated per breeding season per female, including females initiating no nest. We selected females that were continuously monitored during the breeding season. Even if all females were fitted with monitoring devices, technical limitations could cause gaps in their monitoring, which could cause the non-detection 
of nesting attempts. To increase the detectability of nesting attempts, we only selected females that were fitted with VHF and monitored from February to the end of June, with at least one individual sighted every two weeks (resulting in 173 monitoring histories from 141 females from 2002 to 2014).

Trapping of nesting females causes systematic nest abandonment; thus, female trapping and egg collection have a positive effect on overall nesting effort, as they promote replacement clutches. However, replacement clutches are initiated later in the breeding season, affecting the distribution of nest initiation dates. Therefore, for those two parameters, we excluded females from the years where their eggs were collected (see online appendix D).

\section{- Brood survey}

Assessing breeding success after hatching and before fledging in species with nidifugous chicks is difficult. It requires the marking and close monitoring of all individuals; a delicate operation that may affect chick survival at this very sensitive stage of life (Barron et al. 2010). As such, we chose to not mark chicks and instead assessed the fate of broods through the monitoring of brooding females. Therefore, between 2002 and 2014, nesting females fitted with VHF transmitters that successfully hatched at least one egg were then searched for at least once per week. Brood failure was considered for a female at a given monitoring interval if the entire brood disappeared before 60 days of age (Hardouin et al. 2012). In total, we monitored 201 broods from 133 females. A few females $(n=12)$ were captured while rearing chicks, at the nest or after leaving the nest, either to take measurements or to replace the transmitter.

\section{Statistical analyses}

\section{Nest and brood survival analyses}


We used the logistic exposure approach of Shaffer (2004) to estimate the daily nest survival rate (defined as the probability of a living nest to not fail in one day). Egg collections and female trapping cause the end of the nest history and produce an unknown "natural" final fate. Theoretically, in occasional cases where they represent a subset of the sample of nests monitored, nests with an unknown final fate are right truncated at the last date they are known to be active for daily nest survival analysis. In our study, the number of clutches collected was relatively important and biased towards wild-born females. Therefore, nests with an unknown final fate were not representative of the sample of the nests monitored and could lead to an overestimation of the daily nest survival of wild-born females relatively to released females. Thus, we excluded all nests with an unknown final fate from the analysis (following Manolis et al. 2000 and personal communication from Stephen Dinsmore). Finally, as records of periodic nest visits were only standardized beginning in 2003, we discarded data collected prior to 2003.

The same statistical approach was used to study brood survival, with the exception that the exposure period starts at hatching and ends 60 days later for successful broods (mean age at fledging, Hardouin et al. 2012, but see details in Bacon et al. 2017a).

\section{Clutch size, egg volume, nest initiation date and nesting effort}

The nesting effort was analysed using a generalized linear mixed effects model with a Poisson distribution and a log link function. The clutch size was analysed based on ordinal regression using cumulative linked mixed-effects models (CLMMs). Egg volumes and nest initiation dates were analysed using linear mixed-effects models (LMMs) fitted by maximum likelihood with a Gaussian distribution and an identity link function.

\section{Origin and age factor}


We grouped nests into categories based on female origin (wild-born, captive-bred released in autumn and captive-bred released in spring). In addition, we assigned to each nest the age of the nesting female: one-year-old or at least two years old (see online appendix E). For wildborn females, only those captured as juveniles could be aged precisely. A previous study focussing only on released females indicated that one-year-old females had lower breeding performance than older individuals (Bacon et al. 2017a). In addition, the percentage of oneyear-old released females initiating at least one nest is low (8\%, ECWP unpublished data). Based on this information, we assumed that all wild-born females with unknown age were at least two years old at their first identification on a nest.

\section{Adjustment variables}

In monitored animals, events such as trapping and marking are stressful and may have some effect on subsequent behavioural and demographic patterns (e.g. unusual movements, increased mortality, cessation of breeding) once released (Casas et al. 2015, Ponjoan et al. 2008). For nesting female Houbara, trapping causes nest abandonment (direct effect) but may also have a carry-over effect on subsequent breeding performance. For females trapped on live chicks, trapping may have a direct effect on brood survival by affecting either the female or the chicks. Therefore, we considered an intra-seasonal carry-over effect of trapping for the following parameters: clutch size, egg volume, daily nest survival and daily brood survival rate, when measured on the replacement clutch consecutive to the trapping of the female (carry-over trapping). In addition, specifically for the daily brood survival rate, we considered a direct effect of trapping (direct trapping) on the brood when trapping of the females occurred while rearing chicks. Carry-over trapping and direct trapping effects were implemented as binary adjustment variables. To account for the temporal variation of breeding performance values within the breeding season, we included a date covariate (in Julian days, $1^{\text {st }}$ of January $=1$, implemented as the nest initiation date for egg volume and clutch size, as the median date between two nest 
visits for the daily nest survival rate, and as the hatching date of the nest for the daily brood survival rate, Grant \& Shaffer 2012). Finally, we considered temporal variation of the daily survival rate of the nest and brood by including, respectively, a binary factor describing whether the nest was in laying or incubation stage and a continuous covariate indicating the age of the brood since hatching (Grant \& Shaffer 2012).

\section{Model selection}

For each breeding parameter analysis, covariates were rescaled, centralized and standardized by two times the standard deviation (Gelman 2008), and factors with two levels were transformed into binary dummy variables (0-1).

We examined the relationship between the breeding parameters and their corresponding variables by developing sets of candidate models that included additive terms of explanatory variables, quadratic terms of continuous temporal covariates (date ${ }^{2}$ and brood age ${ }^{2}$ ) and the interaction between the age of the nesting female and its origin. To adjust for potential effects of trapping, we kept the trapping carry-over effect and the direct trapping effect in all candidate models of the concerned breeding parameters. Additionally, current year, female identity and nest identity nested under female identity (only for egg volume analyses) were incorporated as random intercept effects in all models. Random intercept effects were included to avoid pseudoreplication and to account for potential heterogeneity among years, females and nests (Zuur et al. 2009).

Thus, the starting (most complex) statistical models were of the following form:

$$
Y=\text { Female Age }+ \text { Female Origin }+ \text { Female Age } \times \text { Female Origin }+ \text { Adjustment variables }
$$

$$
+ \text { random intercepts }
$$


where $Y$ was the dependent variable (the breeding parameter). Details of specific adjustment variables (mentioned in the section Adjustment variables) implemented in the starting model of each breeding parameter can be found in Appendix F.

Starting from this generic structure, models were simplified to develop all possible combinations of explanatory variables (including additive, polynomial terms and the interaction, see all model combinations in Appendix G). This procedure was applied to our six breeding dependent variables. For each breeding dependent variable, we ranked models using the Akaike Information Criterion corrected for small sample size (AICc). The best model $(\triangle \mathrm{AICc}=0$; Burnham \& Anderson 2002) was selected to estimate $\beta$ coefficients and associated 95\% confidence intervals for fixed-effect covariates. All analyses were conducted using R 3.3.3 (R Development Core Team, 2017) and the packages lme4 1.1-12 (Bates et al. 2015), Ordinal 2015.6-28 (Christensen 2015), MuMIn 1.13.4 (Barton 2015), and nest survival (Herzog 2009).

Normality and homoscedasticity of the random residuals were checked graphically from the starting models, and no overdispersion (Zuur et al. 2009) was observed for the Poisson distribution model.

\section{Results}

On average, females were monitored over $2.97 \pm 1.50$ years $(3.58 \pm 1.76$ years for wild-born females and $2.70 \pm 1.25$ years for released females) and $86 \%$ of females were monitored over more than 1 year (88\% for wild-born females and $85 \%$ for released females).

We analysed the effects of dependent covariates on our six breeding parameters based on a common statistical approach. To keep the presentation of the results clear and straightforward, we directly present the coefficients from the best selected models $(\triangle \mathrm{AICc}=0)$ in Table 1 . AICc based model ranking selections, which compiled six tables, can be found in online appendix G. 
According to the best models (Table 1), clutch size and egg volume declined along the breeding season. Daily nest survival was higher during the nest initiation stage than during the incubation stage, while daily brood survival increased with the age of the brood. For all breeding parameters concerned (clutch size, egg volume, daily nest survival and daily brood survival), the carry-over trapping effect had $95 \%$ confidence intervals overlapping 0 , whereas direct trapping of females while rearing chicks had a very strong negative impact on the daily survival of the brood (Table 1). For all breeding parameters (with the exception of the daily nest survival rate), the Age effect was selected in all best models, indicating that one-year-old females had poorer breeding performances than older ones (Table 1).

The origin factor was selected in the best model sets for nesting effort, egg volume and daily brood survival rate. In Table 1, for the female origin factor, the results show the differences with respect to a reference level at the intercept. We defined wild-born females as the reference level at the intercept in Table 1, but all levels of the female origin factor were set as the reference level at the intercept in order to assess potential significant differences. Captive-bred females initiated fewer nests and produced smaller eggs than wild-born females (Figs 3A, B). However, confidence intervals of these effects did not overlap 0 only for captive-bred females released in spring relative to wild-born females (Table 1). Captive-bred females released in autumn had intermediate values of nesting effort and egg volume, but with confidence intervals difference to the two other groups overlapping 0 (Table 1). Finally, captive-bred females released in spring had lower daily brood survival than wild-born females and captive-bred females released in autumn (Fig. 3C, Table 1), and the confidence intervals of the difference between captive-bred females released in spring and wild-born females was close to negative (Table 1).

We were particularly interested in the interaction term of the age of nesting females and their origin, which quantifies whether any effect of origin depends on age (i.e., short-term) or not (i.e., long lasting). However, none of the best models (Table 1) included this interaction, 
providing no support for a more marked effect of the origin in one-year-old females as compared to older females.

\section{Discussion}

The assessment of the breeding performances of released individuals is of major importance in translocation programmes (Bertolero et al. 2009). In this study, we investigated the differences of breeding parameters between wild and captive-bred released Houbara females and revealed how release strategies may influence the magnitude of this differences. This is a prerequisite to adapting and improving translocation strategies and thus to increase the chance of translocation success (Armstrong \& Seddon 2008).

Most breeding parameters (except nest survival) consistently showed age-specific variation, suggesting that one-year-old females have reduced performances compared to older ones, in agreement with a previous study (Bacon et al. 2017a). Individual heterogeneity was taken into account in our statistical framework (see Nussey et al. 2006, van de Pol \& Verhulst 2006). This suggests that the observed effect of age is best explained by longitudinal improvement of individuals (increased breeding experience or an increase in breeding investment, Curio 1983), than by population level processes (e.g., selection of the best individuals over time). The lack of age effects on the daily nest survival may be explained by the weight of external factors, such as environmental conditions (Morales et al. 2002) or predation, which is a prominent cause of nest failure in birds (Martin 1995), including Houbara (Bacon et al. 2017a, Koshkin et al. 2016), potentially overriding any age-dependent effects.

Regardless of age effects, our results indicate that, for some of the breeding parameters studied, wild-born individuals performed slightly better than individuals translocated in autumn and better than individuals translocated in spring. More precisely, wild-born females initiated more nests with larger eggs when compared to captive-bred females released in spring. In addition, 
the daily brood survival of captive-bred females released in spring was lower than for wildborn females. These differences have a marked size effect. It corresponds to a decline of $\sim 47 \%$ in nesting effort and a decline of $\sim 40 \%$ in brood survival (from hatching to fledging) when considering adult females released in spring relative to wild-born adult females (Figs 3A, C).

Conversely to our expectations, we did not observe any interaction between the age of the female and its origin. This result is consistent with the long lasting (i.e., permanent) negative post release effect on breeding parameters observed in other translocated species (see, e.g., Sarrazin et al. 1996). It is also consistent with previous findings in Houbara that released captive-bred females exhibit age-dependent patterns of variation in breeding performance, similar to those observed in many vertebrate populations in the wild (i.e., a bell-shaped trend divided into youth, adulthood and senescence; see discussion in Bacon et al. 2017a).

As differences between wild-born and captive-bred individuals were long lasting, we cannot exclude the possibility that genetic causes related to captive-breeding issues partly explain the observed differences. However, strong and restrictive guidelines are conducted in the ECWP captive breeding programme (Chargé et al. 2014). These guidelines aim to minimize the loss of genetic variation and to minimize the adaptation to captivity in the captive population in order to maximize the fitness of individuals released into the wild, while reducing the genetic consequences of the reinforcement programme on wild populations (Chargé et al. 2014). Nevertheless, the relaxation of sexual and natural selection and adaptation to captivity may partly explain the lower breeding performances of captive-bred individuals in the wild (Araki et al. 2007, Frankham 2008). Beside, owing to the substantial translocation effort (see online appendix B), a substantial proportion of wild-born individuals may be offspring from translocated birds (see general discussion in Barbanera et al. 2010), which may explain why the breeding performances of released and wild-born females are relatively similar. Therefore, it is likely that most differences between the two groups are related to non-genetic issues. 
At the phenotypic level, previous research into captive breeding/rearing in birds has suggested that demographic differences between released and wild-born individuals can be explained by the lack of exposure of chicks hand-reared in captivity to adult behaviour (Roche et al. 2008), permanently decreasing their fitness in the wild (Buner \& Schaub 2008, Buner et al. 2011, Pérez et al. 2015, Sánchez-Garcia et al. 2011). Although wild-born and released captive-bred Houbara females have a similar diet (Bourass \& Hingrat 2015) and exploit equivalent ecological niches (Monnet et al. 2015), released females may be less efficient at finding and exploiting resources (Champagnon et al. 2012). This could explain the lower investment of captive-bred females in reproduction (e.g., egg production), which may subsequently affect the survival of the chicks. In species with precocial chicks (including nidifugous chicks), smaller eggs generally contain less yolk, giving rise to chicks that are in lower condition with lower nutrient reserves at hatching and that are more prone to early mortality (Amundsen \& Stokland 1990, Blomqvist et al. 1997, Galbraith 1988, Williams 1994). The differences observed between wild-born and translocated females must be interpreted while taking into account that all individuals were fitted with transmitters. Markings devices can have significant impacts on some demographic, physiological and behavioural parameters (Barron et al. 2010, see discussion in Le Maho et al. 2014). However, because wild-born and released females are unlikely to exhibit differential responses to transmitter fitting, our main conclusions are unlikely to be affected by transmitter effects.

Our results indicate that the difference observed between wild-born and released females varies according to the period of release (autumn versus spring). Captive-bred females released in autumn had consistently higher nesting effort, egg volume and daily brood survival values than females released in spring (Table 1). Assuming that survival and reproductive parameters positively covary (e.g., "fixed heterogeneity", Cam et al. 2016), such a pattern may be related to the death of the less performant females between their release and the breeding season among 
females released in autumn. This hypothesis is supported by previous findings showing that harsh environmental conditions in autumn and winter lower short-term survival rates compared to spring releases (Hardouin et al. 2014), representing a stronger environmental filter that primarily affects weak individuals.

It is important to note that not all breeding parameters differed between wild-born and captivebred females. The origin of females was not selected in the best models for clutch size, nest initiation date and daily nest survival analysis. Similar heterogeneous responses of multiple breeding parameters to female origin were observed by Hill \& Roberston (1988) for a reinforced ring-necked pheasant (Phasianus colchicus) population, where wild-born and captive-bred released females had similar nesting effort and nesting success, yet chick survival to fledging and total productivity were higher for wild-born females.

\section{Management implications}

We observed consistent, permanent impairments in some breeding parameters of captive-bred released females relative to wild-born females. The magnitude of these differences highlights the importance of assessing demographic impacts of captivity and post release effects in translocated populations. In our study, the observed differences between wild-born and released females appears to be dependent on the translocation strategy (period of release), which implies that it could be buffered by adapting translocation strategies (favouring autumn releases). Nonetheless, the current strategy (spring and autumn releases) seems suitable regarding the multiple objectives of the reinforcement programme, i.e., reinforcement of the wild population and supplementation for hunting (falconry). For autumn releases, environmental stochasticity may select individuals in good condition (Hardouin et al. 2014) that will later breed with relatively better parameters (as suggested by the present results). On the other hand, although outcomes from spring releases will be less certain in terms of breeding performances, higher 
survival rates in this season will help to maintain the population size at a level that can sustain hunting pressure (Hardouin et al. 2015a) and population growth rate. Finally, although differences were observed between wild-born and captive-bred females, our results confirm the capacity of captive-bred females to reproduce in the wild and to contribute to the dynamics of the population beyond their individual history. This partially supports the success of the reinforcement programme (Bertolero \& Oro 2009) and provides a strong basis for more integrative and quantitative assessment of translocation success (Robert et al. 2015a).

\section{Acknowledgements}

This study was funded by the Emirates Center for Wildlife Propagation (ECWP), a project of the International Fund for Houbara Conservation (IFHC). We are grateful to H.H. Sheikh Mohammed bin Zayed Al Nahyan, Crown Prince of Abu Dhabi and Chairman of the IFHC and H.E. Mohammed Al Bowardi, Deputy Chairman of IFHC, for their support. This study was conducted under the guidance of Reneco International Wildlife Consultants LLC., a consulting company managing ECWP. We are thankful to Dr. F. Lacroix, managing director, and G. Leveque, project director, for their supervision. We sincerely thank all ECWP staff from the Ecology division who participated in data collection. We are thankful to Pascale Reding and Grégoire Liénart for producing our study area map. We are grateful to Doug Armstrong and two anonymous reviewers for their helpful and constructive comments on the manuscript, as well as to Dr Thomas Martin for improving the English text.

\section{References}

Amundsen, T., Stokland, J.N., 1990. Egg size and parental quality influence nestling growth in the shag. The Auk 107, 410-413.

Araki, H., Cooper, B., Blouin, M.S., 2007. Genetic Effects of Captive Breeding Cause a Rapid, Cumulative Fitness Decline in the Wild. Science 318, 100-103. 
Armstrong, D.P., Ewen, J.G., 2001. Assessing the value of follow-up translocations: a case study using New Zealand robins. Biological Conservation 101, 239-247. doi:10.1016/S0006-3207(01)00071-4

Armstrong, D.P., Seddon, P.J., 2008. Directions in reintroduction biology. Trends in Ecology \& Evolution 23, 20-25. https://doi.org/10.1016/j.tree.2007.10.003

Armstrong, D.P., Le Coeur, C., Thorne, J.M., Panfylova, J., Lovegrove, T.G., Frost, P.G.H., Ewen, J.G., 2017. Using Bayesian mark-recapture modelling to quantify the strength and duration of post-release effects in reintroduced populations. Biological Conservation 215, 39-45. https://doi.org/10.1016/j.biocon.2017.08.033

Azar, J.F., Rautureau, P., Lawrence, M., Calabuig, G., Hingrat, Y., 2016. Survival of reintroduced Asian houbara in United Arab Emirates' reserves. The Journal of Wildlife Management 80, 1031-1039. doi:10.1002/jwmg.21085

Bacon, L., Hingrat, Y., Robert, A., 2017a. Evidence of reproductive senescence of released individuals in a reinforced bird population. Biological Conservation 215, 288-295. doi:10.1016/j.biocon.2017.08.023

Bacon, L., Hingrat, Y., Jiguet, F., Monnet, A.C., Sarrazin, F., Robert, A., 2017b. Habitat suitability and demography, a time dependent relationship. Ecol. Evol. 2214-2222.

Bacon, L., 2017. Etude des paramètres de reproduction et de la dynamique d'une population renforcée d'outardes Houbara nord-africaines (Chlamydotis undulata undulata) au Maroc. Thèse, Muséum National d'Histoire Naturelle, 192 pp.

Barbanera, F., Pergams, O.R.W., Guerrini, M., Forcina, G., Panayides, P., Dini, F., 2010. Genetic consequences of intensive management in game birds. Biological Conservation 143, 1259-1268. doi:10.1016/j.biocon.2010.02.035 
Barron, D.G., Brawn, J.D., Weatherhead, P.J., 2010. Meta-analysis of transmitter effects on avian behaviour and ecology. Methods in Ecology and Evolution 1, 180-187. doi:10.1111/j.2041-210X.2010.00013.x

Barton, K., 2015. MuMIn: multi-model inference. R package version 1 (9), 13.

Bates, D., M. Martin, B. Bolker, S. Walker., 2015. Fitting Linear Mixed-Effects Models Using lme4. Journal of Statistical Software 67, 1-48 doi:10.18637/jss.v067.i01.

Bertolero, A., Oro, D., Besnard, A., 2007. Assessing the efficacy of reintroduction programmes by modelling adult survival: the example of Hermann's tortoise. Animal Conservation 10, 360-368. doi:10.1111/j.1469-1795.2007.00121.x

Bertolero, A., Oro, D., 2009. Conservation diagnosis of reintroducing Mediterranean pond turtles: what is wrong? Animal Conservation 12, 581-591. doi:10.1111/j.14691795.2009.00284.x

Blomqvist, D., Johansson, O.C., Götmark, F., 1997. Parental quality and egg size affect chick survival in a precocial bird, the lapwing Vanellus vanellus. Oecologia 110, 18-24. doi:10.1007/s004420050128

Bourass, K., Hingrat, Y., 2015. Diet of released captive-bred North-African houbara bustards. European Journal of Wildlife Research 61, 563-574. doi:10.1007/s10344-015-0930-8

Bourass, K., Zaime, A., Qninba, A., Benhoussa, A., Rguibi, H., Hingrat, Y., 2012. Evolution saisonnière du régime alimentaire de l'Outarde houbara nord-africain, Chlamydotis undulata. Bulletin de 1'Instut Scientifique, Rabat 34, 29-43.

Brown, J.L., Collopy, M.W., Gott, E.J., Juergens, P.W., Montoya, A.B., Hunt, W.G., 2006. Wild-reared aplomado falcons survive and recruit at higher rates than hacked falcons in a common environment. Biological Conservation 131, 453-458. doi:10.1016/j.biocon.2006.02.021 
Buner, F., Schaub, M., 2008. How do different releasing techniques affect the survival of reintroduced grey partridges Perdix perdix? Wildlife Biology 14, 26-35. doi:10.2981/0909-6396(2008)14[26:HDDRTA]2.0.CO;2

Buner, F.D., Browne, S.J., Aebischer, N.J., 2011. Experimental assessment of release methods for the re-establishment of a red-listed galliform, the grey partridge (Perdix perdix). Biological Conservation 144, 593-601. doi:10.1016/j.biocon.2010.10.017

Burnham, K.P., Anderson, D.E., 2002. Model selection and multimodel inference - A practical information-theoric approach, Second Edition. Ed. Springer.

Cam, E., Aubry, L.M., Authier, M., 2016. The Conundrum of Heterogeneities in Life History Studies. Trends in Ecology \& Evolution 31, 872-886. doi:10.1016/j.tree.2016.08.002

Casas, F., Benítez-López, A., García, J.T., Martín, C.A., Viñuela, J., Mougeot, F., 2015. Assessing the short-term effects of capture, handling and tagging of sandgrouse. Ibis 157, 115-124. doi:10.1111/ibi.12222

Champagnon, J., Guillemain, M., Elmberg, J., Massez, G., Cavallo, F., Gauthier-Clerc, M., 2012. Low survival after release into the wild: assessing "the burden of captivity" on Mallard physiology and behaviour. European Journal of Wildlife Research 58, 255-267. doi:10.1007/s10344-011-0573-3

Chargé, R., Saint Jalme, M., Lacroix, F., Cadet, A., Sorci, G., 2010. Male health status, signalled by courtship display, reveals ejaculate quality and hatching success in a lekking species. Journal of Animal Ecology 79, 843-850. doi:10.1111/j.1365-2656.2010.01696.x

Chargé, R., Sorci, G., Saint Jalme, M., Lesobre, L., Hingrat, Y., Lacroix, F., Teplitsky, C., 2014. Does recognized genetic management in supportive breeding prevent genetic changes in life-history traits? Evolutionary Applications 7, 521-532. doi:10.1111/eva.12150

Christensen, R. H. B., 2015. ordinal - Regression Models for Ordinal Data. R package version 2015, 6-28 
574 Converse, S.J., Moore, C.T., Armstrong, D.P., 2013. Demographics of reintroduced

575

576

577

578

579

580

581

582

583

584

585

586

587

588

589

590

591

592

593

594

595

596

597

598 populations: Estimation, modelling, and decision analysis. Journal of Wildlife Management 77, 1081-1093. https://doi.org/10.1002/jwmg.590

Curio, E., 1983. Why young birds reproduce less well? Ibis 125, 400-404. doi:10.1111/j.1474919X.1983.tb03130.x

Dickens, M.J., Delehanty, D.J., Michael Romero, L., 2010. Stress: An inevitable component of animal translocation. Biological Conservation 143, 1329-1341. doi:10.1016/j.biocon.2010.02.032

Ewen, J.G., Armstrong D.P., Parker K.A., Seddon, P.J., 2012. Reintroduction Biology: Integrating Science and Management. Wiley-Blackwell

Fischer, J., Lindenmayer, D.B., 2000. An assessment of the published results of animal relocations. Biological Conservation 96, 1-11. doi:10.1016/S0006-3207(00)00048-3

Frankham, R., 2008. Genetic adaptation to captivity in species conservation programs. Molecular Ecology 17, 325-333. doi:10.1111/j.1365-294X.2007.03399.x

Galbraith, H., 1988. Effects of egg size and composition on the size, quality and survival of lapwing Vanellus vanellus chicks. Journal of Zoology 214, 383-398. doi:10.1111/j.14697998.1988.tb03747.x

Gaucher, P., 1995. Breeding biology of the houbara bustard Chlamydotis undulata in Algeria. Alauda 63, 291-298.

Gelman, A., 2008. Scaling regression inputs by dividing by two standard deviations. Statistics in medicine 27, 2865-2873.

Goriup, P. D., 1997. The world status of the houbara bustard Chlamydotis undulata. Bird Conservation International 7, 373-397.

Grant, T.A., Shaffer, T.L., 2012. Time-specific patterns of nest survival for ducks and passerines breeding in North Dakota. The Auk 129, 1-10. 
Hardouin, L.A., Nevoux, M., Robert, A., Gimenez, O., Lacroix, F., Hingrat, Y., 2012. Determinants and costs of natal dispersal in a lekking species. Oikos 121, 804-812. doi:10.1111/j.1600-0706.2012.20313.x

Hardouin, L.A., Robert, A., Nevoux, M., Gimenez, O., Lacroix, F., Hingrat, Y., 2014. Meteorological conditions influence short-term survival and dispersal in a reinforced bird population. Journal Applied Ecology 51, 1494-1503. doi:10.1111/1365-2664.12302

Hardouin, L.A., Hingrat, Y., Nevoux, M., Lacroix, F., Robert, A., 2015a. Survival and movement of translocated houbara bustards in a mixed conservation area. Animal conservation, 18(5), 461-470.

Hardouin, L.A., Legagneux, P., Hingrat, Y., Robert, A., 2015b. Sex-specific dispersal responses to inbreeding and kinship. Animal Behaviour 105, 1-10. doi:10.1016/j.anbehav.2015.04.002

Herzog, M., 2009. nestsurvival. R package that performs logistic exposure nest survival analyses.

Hill, D., Robertson, P., 1988. Breeding Success of Wild and Hand-Reared Ring-Necked Pheasants. The Journal of Wildlife Management 52, 446-450. doi:10.2307/3801588

Hoyt, D., 1979. Practical methods of estimating volume and fresh weight of birg eggs. The Auk $96,73-77$.

Huff, D.D., Miller, L.M., Chizinski, C.J., Vondracek, B., 2011. Mixed-source reintroductions lead to outbreeding depression in second-generation descendents of a native North American fish. Molecular Ecology 20, 4246-4258. doi:10.1111/j.1365294X.2011.05271.x

IUCN/SSC. 2013. Guidelines for reintroductions and other conservation translocations. Version 1.0. Gland, Switzerland. Available from http://www.issg.org/pdf/publications/RSG_ISSG-Reintroduction-Guidelines-2013.pdf 
Koshkin, M., Burnside, R.J., Packman, C.E., Collar, N.J., Dolman, P.M., 2016. Effects of habitat and livestock on nest productivity of the Asian houbara Chlamydotis macqueenii in Bukhara Province, Uzbekistan. European Journal Wildlife Research 62, 447-459. doi:10.1007/s10344-016-1018-9

Lacroix, F., Seabury, J., Al Bowardi, M., and Renaud, J., 2003. The Emirates Center for Wildlife Propagation: comprehensive strategy to secure self-sustaining wild populations of houbara bustard (Chlamydotis undulata undulata) in Eastern Morocco. Houbara News $5,60-62$.

Le Gouar, P., Robert, A., Choisy, J.-P., Henriquet, S., Lecuyer, P., Tessier, C., Sarrazin, F., 2008. Roles of Survival and Dispersal in Reintroduction Success of Griffon Vulture (Gyps fulvus). Ecological Applications 18, 859-872. doi:10.1890/07-0854.1

Le Maho, Y., Whittington, J.D., Hanuise, N., Pereira, L., Boureau, M., Brucker, M., Chatelain, N., Courtecuisse, J., Crenner, F., Friess, B., Grosbellet, E., Kernaléguen, L., Olivier, F., Saraux, C., Vetter, N., Viblanc, V.A., Thierry, B., Tremblay, P., Groscolas, R., Le Bohec, C., 2014. Rovers minimize human disturbance in research on wild animals. Nature Methods 11, 1242-1244. https://doi.org/10.1038/nmeth.3173

Lesobre, L., Lacroix, F., Caizergues, A., Hingrat, Y., Chalah, T., Jalme, M.S., 2010. Conservation genetics of Houbara Bustard (Chlamydotis undulata undulata): population structure and its implications for the reinforcement of wild populations. Conservation Genetics 11, 1489-1497. doi:10.1007/s10592-009-9979-9

Mabee, T.J., 1997. Using Eggshell Evidence to Determine Nest Fate of Shorebirds. The Wilson Bulletin 109, 307-313. doi:10.1111/j.1557-9263.2006.00037.x

Manolis, J.C., Andersen, D.E., Cuthbert, F.J., 2000. Uncertain nest fates in songbird studies and variation in mayfield estimation. The Auk 117, 615-626. doi:10.1642/00048038(2000)117[0615:UNFISS]2.0.CO;2 
Martin, T.E., 1995. Avian Life History Evolution in Relation to Nest Sites, Nest Predation, and Food. Ecological Monographs 65, 101-127. doi:10.2307/2937160

Mauck, R.A., Huntington, C.E., Doherty Jr, P.F., 2012. Experience versus effort: what explains dynamic heterogeneity with respect to age? Oikos 121, 1379-1390. doi:10.1111/j.16000706.2012.20271.x

Mayfield, H.F., 1975. Suggestions for Calculating Nest Success. The Wilson Bulletin 87, 456466. doi: $10.2307 / 4160682$

Mihoub, J.-B., Robert, A., Gouar, P.L., Sarrazin, F., 2011. Post-Release Dispersal in Animal Translocations: Social Attraction and the "Vacuum Effect". PLOS ONE 6, e27453. doi:10.1371/journal.pone.0027453

Monnet, A.-C., Hingrat, Y., Jiguet, F., 2015. The realized niche of captive-hatched Houbara Bustards translocated in Morocco meets expectations from the wild. Biological Conservation 186, 241-250. doi:10.1016/j.biocon.2015.03.013

Montalvo, A.M., Ellstrand, N.C., 2001. Nonlocal transplantation and outbreeding depression in the subshrub Lotus scoparius (Fabaceae). American Journal of Botany 88, 258-269.

Morales, M.B., Alonso, J.C., Alonso, J., 2002. Annual productivity and individual female reproductive success in a Great Bustard Otis tarda population. Ibis 144, 293-300. doi:10.1046/j.1474-919X.2002.00042.x

Nussey, D.H., Kruuk, L.E.B., Donald, A., Fowlie, M., Clutton-Brock, T.H., 2006. The rate of senescence in maternal performance increases with early-life fecundity in red deer. Ecology Letters 9, 1342-1350. doi:10.1111/j.1461-0248.2006.00989.x

Pérez, J.A., Sánchez-García, C., Díez, C., Bartolomé, D.J., Alonso, M.E., Gaudioso, V.R., 2015. Are parent-reared red-legged partridges (Alectoris rufa) better candidates for reestablishment purposes? Poultry Science 94, 2330-2338. doi:10.3382/ps/pev210 
Ponjoan, A., Bota, G., De La Morena, E.L.G., Morales, M.B., Wolff, A., Marco, I., Mañosa, S., 2008. Adverse Effects of Capture and Handling Little Bustard. The Journal of Wildlife Management 72, 315-319. doi:10.2193/2006-443

R Development Core Team, 2017. R: A language and environment for statistical computing. R Foundation for Statistical Computing, Vienna, Austria.

Robert, A., Couvet, D., Sarrazin, F., 2007. Integration of demography and genetics in population restorations. Ecoscience 14, 463-471. doi:10.2980/11956860(2007)14[463:IODAGI]2.0.CO;2

Robert, A., 2009. Captive breeding genetics and reintroduction success. Biological Conservation 142, 2915-2922. doi:10.1016/j.biocon.2009.07.016

Robert, A., Colas, B., Guigon, I., Kerbiriou, C., Mihoub, J.-B., Saint-Jalme, M., Sarrazin, F., 2015a. Defining reintroduction success using IUCN criteria for threatened species: a demographic assessment. Animal Conservation 18, 397-406. doi:10.1111/acv.12188

Robert, A., Bolton, M., Jiguet, F., Bried, J., 2015b. The survival-reproduction association becomes stronger when conditions are good. Proceedings of the Royal Society B 282, 20151529. doi:10.1098/rspb.2015.1529

Roche, E.A., Cuthbert, F.J., Arnold, T.W., 2008. Relative fitness of wild and captive-reared piping plovers: Does egg salvage contribute to recovery of the endangered Great Lakes population? Biological Conservation 141, 3079-3088. doi:10.1016/j.biocon.2008.09.014

Saint Jalme, M., Van Heezik, Y., 1996. Propagation of the houbara bustard. Paul Keegan, London.

Sánchez-García, C., Alonso, M.E., Pérez, J.A., Rodríguez, P.L., Gaudioso, V.R., 2011. Comparing fostering success between wild-caught and game farm bred captive redlegged partridges (Alectoris rufa, L.). Applied Animal Behaviour Science 133, 70-77. doi:10.1016/j.applanim.2011.04.012 
Sarrazin, F., Bagnolini, C., Pinna, J.L., Danchin, E., Clobert, J., 1994. High Survival Estimates of Griffon Vultures (Gyps fulvus fulvus) in a Reintroduced Population. The Auk 111, 853-862. doi:10.2307/4088817

Sarrazin, F., Bagnolinp, C., Pinna, J.L., Danchin, E., 1996. Breeding biology during establishment of a reintroduced Griffon Vulture Gyps fulvus population. Ibis 138, 315325. doi:10.1111/j.1474-919X.1996.tb04344.x

Shaffer, T.L., 2004. A unified approach to analyzing nest success. The Auk 121, 526-540.

Sutherland, W.J., Armstrong, D., Butchart, S.H.M., Earnhardt, J.M., Ewen, J., Jamieson, I., Jones, C.G., Lee, R., Newbery, P., Nichols, J.D., Parker, K.A., Sarrazin, F., Seddon, P.J., Shah, N., Tatayah, V., 2010. Standards for documenting and monitoring bird reintroduction projects. Conservation Letters 3, 229-235. doi:10.1111/j.1755263X.2010.00113.x

Tavecchia, G., Viedma, C., Martínez-Abraín, A., Bartolomé, M.-A., Gómez, J.A., Oro, D., 2009. Maximizing re-introduction success: Assessing the immediate cost of release in a threatened waterfowl. Biological Conservation 142, 3005-3012. doi:10.1016/j.biocon.2009.07.035

van de Pol, M., Verhulst, S., 2006. Age- Dependent Traits: A New Statistical Model to Separate Within- and Between- Individual Effects. The American Naturalist 167, 766-773. doi:10.1086/503331

Williams, T.D., 1994. Intraspecific variation in egg size and egg composition in birds: effects on offspring fitness. Biological Reviews of the Cambridge Philosophical Society 69, 3559.

Zuur, A.F., Ieno, E.N., Walker, N., Saveliev, A.A., Smith, G.M., 2009. Mixed effects models and extensions in ecology with R, Statistics for Biology and Health. Springer New York, New York, NY. doi:10.1007/978-0-387-87458-6 
